
DK 678.675'126:547.391.1:[678.9 - 02:66.095.26]: 541.124.2:546.714:

\section{Teichmann, Rainer}

Polyamid 6 als Rückgratpolymeres bei mangan(IV)initiierten Pfropfpolymerisationen

Faserforsch. u. Textiltechnik 24 (1973) 9, S. 351-354. 2 (3) Abb., 2 Tab., 13 Lit.

Es werden einige Untersuchungen zum Verlauf und zur Wirksamkeit der mangan(IV)-initiierten Pfropfpolymerisation auf Polyamid 6 geschildert, wobei die Hôhe der Pfropfausbeuten in Abhăngigkeit von den Versuchsbedingungen im Vordergrund steht. Als praktisches Anwendungsbeispicl dient eine Antistatikausrüstung, realisiert durch Pfropfung von Polyamid-6Faserstoff mit Polyacrylsāure und Ùberführung der polymeren Săure in ihr Ammoniumsalz.
DK 661.728:677.463:677.021.122.223:677.021.122.225:541.128.34:546.171.1 Schleicher, Harry, Daniels, Carola, und Philipp, Burkart

Zum Einfluß einer Aktivierung von Cellulose mit Ammoniak auf deren anschließende Alkalisierung und Xanthogenierung Faserforsch. u. Textiltechnik 24 (1973) 9, S. 371-376. 7 Abb., 3 Tab., 14 Lit.

Nach einer Aktivierung von Cellulose mit flüssigem Ammoniak oder wåßrigen Ammoniaklösungen erfolgt im Vergleich zur unbehandelten Celluse die Cmwandlung zu Natroncellulose I bzw. die Auflosung bei eine trations wesenthich niearigeren NaOH-Konzenvation, wober sich allerdings das Linwand Loseintervall

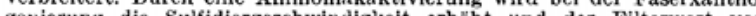
genierung die Sulfidic en verbessert.
DK $677.521: 677.019 .34: 677.017 .635 .3: 677.017 .635 .2: 677.014 .8$

\section{Wiedemann, Gottfried, und Frenzel, Horst}

Untersuchungen zur chemischen Beständigkeit der Glasseide Faserforsch. u. Textiltechnik 24 (1973) 9, S. 355-359. 12 Abb., 3 Tab., 9 Lit.

Einige durch Einwirkung von Wasser, Mineralsãuren und Alkali bewirkte Verånderungen der Glasseidenstruktur und -oberflăche von alkaliarmer und alkalireicher (Glasseide wurden untersucht. Während Alkali die Glasseidenstruktur völlig zerstört, werden bei Mineralsäurebeanspruchungen in Abhăngigkeit vom Thermobehandlungs\%ustand selektiv Netzwerkwandler herausgelőst.
DK $677.494 .745 .32: 677.014 .845: 539.25: 677.014 .87: 677.019 .2: 532.528$

Walenta, Ernst, und Teichgräber, Michael

Zur Bestimmung der Hohlraumstruktur von PVY-Faserstoffen mittels Röntgenkleinwinkelstreuung

Faserforsch. u. Textiltechnik 24 (1973) 9, S. 360-364. 5 Abb., 3 Tab., 20 lit.

Die Röntgenkleinwinkelstreuung (RKWS) hat für die Untersuchung der Hohlraumstruktur von Polymeren zunehmend Bedentung erlangt. Im Folgenden wird ûber die Bestimmung der Größenverteilung der Hohlrăume in PVY-Modellfäden auf der Grundlage eines früher vorgeschlagenen Verfahrens berichtet. Durch vergleichende elektronenmikroskopische Aufnahmen wird sichergestellt, daB es sich bei der RKWS um eine Hohlratmstreuung handelt. DK $547.584^{\prime} 023: 678.674^{\prime} 524^{\prime} 420: 677.494 .674^{\prime} 524^{\prime} 420: 543.283 .062:$

Berger, Werner, und Ewert, Karl-Heinz

Über das polarographische Verhalten einiger Derivate der Terephthal- und Isophthalsäure

2. Mitt.: Die selektive Bestimmung von Terephthalsäureestergliedern in Polymeren und Polymerkombinationen

Faserforsch. u. Textiltechnik 24 (1973) 9, S. 377-380. 1 Tab., 28 Lit.

Die Abspaltung von Terephthalsäureestereinheiten durch Hydrazin und die selektive polarographische Bestinmung des bei der spaltung entstchenden Terephthalsăuredihydrazids wird beschrieben. Diese spezifische Spaltungsreaktion und die polarographische Reduzierbarkeit des Hydrazinolyschauptproduktes bilden die Grundlage zu einer neuen quantitativen Bestimmungsmethode für Terephthalsaurcanteile in listern. Dazu zăhle und Bikomponenten, Polymermischungen sowic Filamentmischgarne

DK $547.584^{\prime} 261: 547.422 .22: 542.951 .1: 541.127 .1: 541.128 .1: 678.674^{\prime} 524^{\prime} 420$ Dimov, Kiril, Terlemesjan, Kkaterina, und Petrov, Peter

Zur Umesterung von Dimethylterephthalat mit Äthylenglykol

laserforsch. u. Textiltechnik $\mathbf{2 4}$ (1973) $9, \quad$ \$. $381-383$. 3 Abb., 2 Tab., 7 Lit.

Die Konzentration verschiedener Produkte der Umesterungsreaktion des Dimethylterephthalats mit Åthylenglykol in Abhãngigkeit von der Reaktionstemperatur, der Konzentration des Katalysators und dem Verhăltnis der Reaktanten wurde untersucht, wobei man sich der statistischen Versuchsplanung bediente. Die erhaltenen Jirgebnisse sind als Gleichungen zweiter Ordunng dargestellt.
DK 678.746.222 - 134.532:66.062:541.183.03:541.183.2:541.183.26

\section{Glöckner, Gottfried, und Lohmann, Dietmar}

Zum Einfluß der Struktur von Copolymeren aut ihre Wechselwirkung mit Lösungsmitteln

Faserforsch. u. Textiltechnik 24 (1973) 9, S. 365-370. 7 Abb., 1 Tab., 43 I.it.

Mit einer Apparatur nach Prager und Long wurde dic Lösungsmittel aufnahme aus der Gasphase von Styrol/Acrylnitril-Copolymeren untersucht. Diese Sorption hängt von strukturellen Parametern ab, die durch die Vorgeschichte der untersuchten Proben bedingt werden. Weiterhin wird auf den Zusammenhang zwischen Lősungsmittelaufnahme im Sorptionsgleichgewicht und der Mikrostruktur des Copolymeren eingegangen. Die erhaltenen Ergebnisse werden mit Hilfe der Flory/Huggins-Theorie ausgewertet. lis ergibt sich, da $B$ der Wechselwirkungsparameter in Abhāngigkeit von der chemischen Zusammensetzung der untersuchten Proben ein deutliches Minimum aufweist.
Neue Bücher S. $384-387$.

Patentschau S. $387-389$

Literatursehau S. $389-392$. 


\title{
Reaktionstypen in der anorganischen Chemie
}

1973. Etwa 368 Seiten - 98 Abbildungen - 105 Tabellen $-8^{\circ}-$ Leinen $35,-M$

Bestell-Nr. 7613509 (5734)

\begin{abstract}
Vom Gesichtspunkt der chemischen Bindung mit Hilfe der Thermodynamik und Kinetik werden die Gesetzmäßigkeiten von anorganisch-chemischen Reaktionen in wäßriger Lösung erarbeitet.

Die Vorausmöglichkeiten für chemische Reaktionen auf der Grundlage von thermodynamischen und kinetischen Faktoren werden zunächst im allgemeinen dargestellt.

Chemische Reaktionen in wäßriger Lösung, die sich auf drei Reaktionstypen reduzieren lassen, Komplexreaktionen, Redoxreaktionen und Säure-BaseReaktionen, werden skizziert.

Im besonderen werden die sich ergebenden Gemeinsamkeiten in der Begriffsbildung und im Wesen der als Teilchenübertragungsreaktionen aufzufassenden Reaktionstypen herausgestellt. Dabei wird die Tendenz, die chemische Reaktionsfähigkeit durch einen einzigen, umfassenden Reaktionstyp zu beschreiben, aufgezeigt.
\end{abstract}

Bestellungen durch eine Buchhandlung erbeten

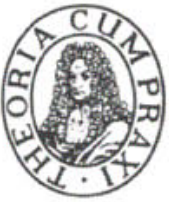

A K A D E M I E - V E R L A G

DDR - 108 Berlin, Leipziger Str. 3-4

Die Zeitschrift „Faserforschung und Textiltechnik“" erscheint monatlich in Heften zu 48 Textsciten im Format A 4 . Der Preis für das Einzelheft beträgt

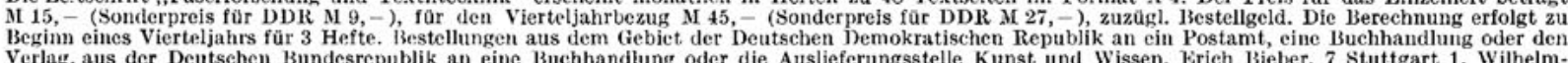
Verlag, aus der Deutschen Bundesrepublik an eine Buchhandlung oder die Auslieferungsstelle Kunst und Wissen, Erich Bieber, 7 Stuttgart 1, Wilhelmstraßc 4-6, ans dem Ausland an eine Importbuchhandlung, den Deutschen Buch-Export und -Import GmbH., 701 Leipzig, PostschlieBfach 276, oder den Heftes: 1014/24/9. Alleinige Anzeigenannahme DEWAG-WERBV NG, 1054 Berlin, Wilhelm-Pieck-Str. 49, und alle DEWAG-Betriebe in den Bezirkstädten der DDR. - Bestellungen in der UdSSR nehmen entgegen: Stüdtische Abteilungen von ,SOJUZPECHATJ“ bzw. Postumter und Postkontore.

Herausgeber und verantwortlich für den Inhalt: Prof. Dr. Dr. Erich Correns, Institut für Polymerenchemie der Akademie der Wissenschaften der DDR, 153 Teltow-Scchof, Fernruf: Teltow 4831 ; Prof. Dr.-Ing. habil. Wolfgang Bobeth, Institut für Technologie der Fasern der Akademie der Wissenschaften der DDR, 801 Dresden, Hohe Str. 6, Fernruf: 44721; Prof. Dr.-Ing. Hans Böhringer, 682 Rudolstadt; Prof. Dr. Hermann Klare, Prof. Dr. habil. Burkart Philipp und Dr. habil. Christian kuscher, Institut für Polymerenchemie der Akademie der Wissenschaften der DDR, 153 Teltow-Seehof, Fernruf: Teltow 4831. Schriftleiter: Joachim Brämer und Dipl.-Chem. Ingeborg Ruscher, 153 Teltow-Scehof, Kantstr. 55. Verlag: Akademie-Verlag GmbH., 108 Berlin, Leipziger
Straße 3-4. Satz und Druck: Druckhaus, ,Maxim Gorki ${ }^{*}, 74$ Altenburg. - Veröffentlicht unter der Lizenznummer 1280 des Presseamtes beim Vorsitzenden des Ministerrates der Deutschen Demokratischen Republik.

Manuskriptscndungen sind an einen der Herausgeber oder die Schriftleitung zu richten. Für Inhalt und Form gelten die „,Richtlinien für die Annahme und Abfassungen von Beiträgen", erhältlich von der Schriftleitung. Die Verfasser gröBerer wisscnschaftlicher Arbeiten erhalten außer dem Honorar ein Heft und 50 Sonderdrucke ihrer Arbeit unentgeltlich. Nachdrucke sowic Übersetzungen in fremde Sprachen des Inhalts dieser Zeitschrift und deren Verbreitung - auch auszugsweise mit Quellenangabe - bedúrfen der schriftlichen Vereinbarung mit dem Verlag. 